\title{
Entanglement Detection
}

\section{with Non-Ideal Ferromagnetic Detectors}

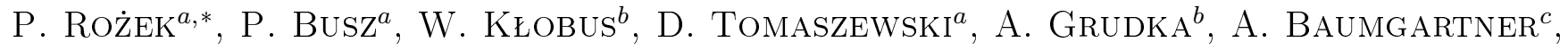 \\ C. SCHÖNENBERGER ${ }^{c}$ AND J. MARTINEK ${ }^{a}$ \\ ${ }^{a}$ Institute of Molecular Physics, Polish Academy of Science, 60-179 Poznan, Poland \\ ${ }^{b}$ Faculty of Physics, Adam Mickiewicz University, 61-614 Poznan, Poland \\ ${ }^{c}$ Department of Physics, University of Basel, CH-4056 Basel, Switzerland
}

\begin{abstract}
Entangled states are essential in basics quantum communication protocols and quantum cryptography. Ferromagnetic contacts can work as a spin detector, giving possibility of converting information about electron spin to the electric charge, and therefore, detection of entangled states with the electric current measurements is possible. Method of confirming entanglement with non-ideal detectors is presented, the impact of decoherence and noise on states and quality of entanglement is discussed. Entanglement witness (EW) operator method is compared with the CHSH inequalities approach. Required spin polarization for the EW is lower than for the CHSH inequalities. System with asymmetric spin polarizations of detectors was analyzed, including the CHSH inequalities and the EW method.
\end{abstract}

DOI: 10.12693 /APhysPolA.127.493

PACS: 03.67.Mn, 03.67.Bg, 73.23.-b

\section{Introduction}

Quantum entanglement is a basic ingredient of quantum communication protocols. Characterization of generated entangled states is an important issue in applications, due to non-ideality of detectors and noise in generated states.

In general, the entanglement can be considered as a correlation that arises from interaction between two quantum subsystems. Subsystems are, however, correlated on any distances what posed a fundamental problem in understanding of quantum mechanics - correlations should be non-local, in opposition to classical view or can be explained in terms of the local hidden variable theory (LHV). Great number of experiments confirmed non-local nature of entanglement and therefore, quantum mechanics [1]. Non-local character of entangled states can be confirmed by the CHSH inequalities violation check. If specific combination of correlation functions exceeds classical limit, states can be considered as correlated non-locally. However, in some applications, only non-separability of states is important, and confirmation of non-locality don't play a crucial role. The maximally entangled states can be defined for two-spin system (Bell states) as:

$$
\begin{aligned}
& \left|\psi_{A B}^{ \pm}\right\rangle=\frac{1}{\sqrt{2}}\left(\left|\uparrow_{A} \downarrow_{B}\right\rangle \pm\left|\downarrow_{B} \uparrow_{A}\right\rangle\right), \\
& \left|\phi_{A B}^{ \pm}\right\rangle=\frac{1}{\sqrt{2}}\left(\left|\uparrow_{A} \uparrow_{B}\right\rangle \pm\left|\downarrow_{B} \downarrow_{A}\right\rangle\right) .
\end{aligned}
$$

Prepared state, due to interactions with reservoir, is

\footnotetext{
* corresponding author; e-mail: piotrrozek91@gmail.com
}

mixed with white noise from environment and can be represented as the Werner state:

$$
\rho_{A, B}^{W}(\lambda)=\lambda|\psi\rangle\langle\psi|+(1-\lambda) \mathbb{I} / 4,
$$

where $A, B$ are two subsystems, $|\psi\rangle$ is one of the Bell states, $\lambda$ is visibility parameter $(\lambda=0$ white noise, $\lambda=1$ pure state) and $\mathbb{I}$ is identity operator. Werner states, depending on the value of visibility parameter, can be considered separable or not.

Fundamental conclusion is that for the Werner state with $\lambda>\frac{1}{3}$ the entanglement can the detected [2]. It can be shown by introducing the entanglement witness (EW) operator method. Separability criterion for density matrices [3] gives possibility for defining an observable that provides information only about separability of state and no information about non-locality. Entanglement witness (EW) operator can be defined in the following way:

$$
\mathbb{W}=\left(\left|\phi_{-}\right\rangle\left\langle\phi_{-}\right|\right)^{T_{B}},
$$

where $\left|\phi_{-}\right\rangle$is a projection operator onto a negative eigenvalue of partially transposed density matrix of the system:

$$
\begin{aligned}
& \rho_{A, B}^{T_{B}}=\left(\sum_{i, j, k, l} c_{i, j, k, l}|i\rangle\left\langle\left. j\right|_{A} \otimes \mid k\right\rangle\left\langle\left. l\right|_{B}\right)^{T_{B}}=\right. \\
& \sum_{i, j, k, l} c_{i, j, k, l}|i\rangle\left\langle\left. j\right|_{A} \otimes \mid l\right\rangle\left\langle\left. k\right|_{B} .\right.
\end{aligned}
$$

For two spins system, the EW for the singlet state can be given by:

$$
\mathbb{W}_{3 D}=\frac{1}{2}\left(\mathbb{I}+\sum_{i=x, y, z} \hat{\sigma}_{i} \otimes \hat{\sigma}_{i}\right)
$$

where $\hat{\sigma}_{i}$ is $i$-component of the Pauli matrix. Therefore, three correlation measurements are required to confirm non-separability of state. The EW can be reduced to 
two-dimensional (2D) case:

$$
\mathbb{V}_{i j}=\frac{1}{2}\left(\mathbb{I}+\hat{\sigma}_{i} \otimes \hat{\sigma}_{i}+\hat{\sigma}_{j} \otimes \hat{\sigma}_{j}\right)
$$

When considering the value of entanglement witness in a specific state, information about separability is given by the sign of average value: generally, an observable can be considered as an entanglement witness if for some entangled state a condition

$$
\langle\mathbb{W}\rangle \equiv \operatorname{tr}\left(\rho_{A, B} \mathbb{W}\right)<0
$$

is satisfied and, for all separable states

$$
\langle\mathbb{W}\rangle \equiv \operatorname{tr}\left(\rho_{A, B} \mathbb{W}\right) \geq 0
$$

is satisfied. These considerations can be applied to a system with generator of two-spins entangled states (eg. superconducting electrode) [4], and two transport channels with non-ideal detectors. a)

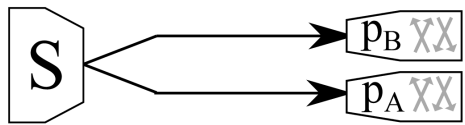

b)

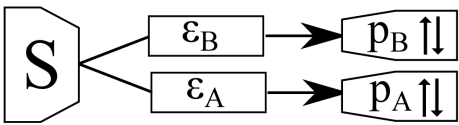

Fig. 1. Considered system with equivalent configuration. Additional depolarization channels with ideal detectors are equivalent to non-ideal detectors. a) shows the situation when in the system detectors are non-ideal; b) shows the equivalent configuration - however here, detectors are ideal but in each transport channel electrons spins are interfered by depolarizing channel..

\section{Ferromagnetic detectors}

In case of electrons, spin measurements can be performed with electric current measurements using magnetic electrodes. Efficiency of the detectors can be expressed as the spin polarization $p_{\eta}$, which is related to density of states at Fermi level $\rho_{\eta, \pm}$ for majority $\left(\rho_{\eta,+}\right)$ and minority $\left(\rho_{\eta,-}\right)$ charge carriers.

$$
p_{\eta}=\frac{\rho_{\eta,+}-\rho_{\eta,-}}{\rho_{\eta,+}+\rho_{\eta,-}},
$$

where $\eta$ denotes channels $A, B$.

In order to describe measurements with non-ideal ferromagnetic detectors, firstly, von Neumann measurements described by projection operators have to be introduced

$$
\hat{\mathcal{P}}_{i}=|i\rangle\langle i|
$$

where measurements are ideal. The probability of the state $i=\{\uparrow, \downarrow\}$ is given by $p_{i}=\operatorname{tr}\left(\hat{\rho} \hat{\mathcal{P}}_{i}\right)$, where $\hat{\rho}$ is a density matrix, and $z$ spin component can be obtained using the operator $\hat{\sigma}_{z}=(1)|\uparrow\rangle\langle\uparrow|+(-1)| \downarrow\rangle\langle\downarrow|$. When detectors are non-ideal, the effective spin polarization is $p<1$, the measurement operators Eq. (10), have to be generalized. State $|\uparrow\rangle$ can be also detected as a state $|\downarrow\rangle$ with some probability. Measurements can be generalized by the POVM (positive operator valued measure) operators $\hat{M}_{i}$, that are positive and form a complete set $\sum_{i} \hat{M}_{i}=\mathbb{I}$. The POVM for single spin can be postulated $\sum_{i}$

$$
\begin{aligned}
& \hat{M}_{\uparrow}=\xi_{+}|\uparrow\rangle\left\langle\uparrow\left|+\xi_{-}\right| \downarrow\right\rangle\langle\downarrow|, \\
& \hat{M}_{\downarrow}=\xi_{+}|\downarrow\rangle\left\langle\downarrow\left|+\xi_{-}\right| \uparrow\right\rangle\langle\uparrow|,
\end{aligned}
$$

where $\xi_{ \pm}$are related to density of states at the Fermi level in a ferromagnetic detector: $\xi_{ \pm} \equiv \frac{\rho_{ \pm}}{\rho_{ \pm}+\rho_{\mp}}=\frac{1}{2}(1 \pm p)$. Therefore, $z$ spin component can be given by:

$$
\hat{\sigma}_{z}^{p}=\hat{M}_{\uparrow}-\hat{M}_{\downarrow}=p \hat{\sigma}_{z} .
$$

Non-ideal measurements can be considered to be an equivalent with depolarizing channel as depicted in
Fig. 1. The depolarizing channel operator for single-qubit system can be defined as:

$$
\varepsilon(\hat{\rho})=p \hat{\rho}+\frac{1}{2}(1-p) \mathbb{I},
$$

where $p$ is a detector efficiency that corresponds to the spin polarization and density matrix $\hat{\rho}$ of state before depolarization. It can be shown that for any observable $\hat{O}$ and density matrix $\hat{\rho}[5]$

$$
\operatorname{tr}(\epsilon(\hat{\rho}) \hat{O})=\operatorname{tr}(\hat{\rho} \epsilon(\hat{O})) .
$$

Depolarized von Neumann measurement projection operators are an equivalent to POVM measurements [5]:

$$
\varepsilon\left(\hat{\mathcal{P}}_{i}\right)=\hat{M}_{i}
$$

and therefore

$$
\operatorname{tr}\left(\epsilon(\hat{\rho}) \hat{\mathcal{P}}_{i}\right)=\operatorname{tr}\left(\hat{\rho} \hat{M}_{i}\right) .
$$

\section{Entanglement witness method}

The EW operator method allows for detection, which states can be considered as entangled. Taking the depolarized Werner state:

$$
\epsilon_{A} \otimes \epsilon_{B} \rho_{A, B}^{W}(\lambda)=\rho_{A, B}^{W}\left(\lambda p_{A} p_{B}\right),
$$

where $\epsilon_{\eta}$ denotes the depolarizing channel $\eta$, and $\rho_{A, B}^{W}(\lambda)$ denotes the Werner state defined in Eq. (18). Here, asymmetry of detectors efficiencies is assumed $\left(p_{A} \neq p_{B}\right)$. The EW can be applied to the considered state. Assuming the Werner state in Eq. (18) consisted of the singlet state and noise, $2 \mathrm{D}(6)$ and $3 \mathrm{D}(5)$ the EWs give average values:

$$
\begin{aligned}
& \operatorname{tr}\left(\rho_{A, B}^{W}\left(\lambda p_{A} p_{B}\right) \mathbb{W}_{3 D}\right)=\frac{1}{2}\left(1-3 \lambda p_{A} p_{B}\right), \\
& \operatorname{tr}\left(\rho_{A, B}^{W}\left(\lambda p_{A} p_{B}\right) \mathbb{V}_{i j}\right)=\frac{1}{2}\left(1-2 \lambda p_{A} p_{B}\right) .
\end{aligned}
$$

States are entangled if averages (19) are negative. For CHSH inequalities required efficiency of detectors $p_{A}, p_{B}$ and visibility of state $\lambda$ is given by:

$$
1-\sqrt{2} \lambda p_{A} p_{B}<0 \text {. }
$$

For strong asymmetry in detectors efficiency, entanglement can also be detected: for example, assume that $\lambda=1$, when $p_{A}=1$ only $p_{B}=\frac{1}{3}$ is required to detect 
entanglement with $\mathbb{W}_{3 D}$, see Fig. 2. These results can be useful in experiments - use of asymmetric and non-ideal detectors can simplify the experimental setup.

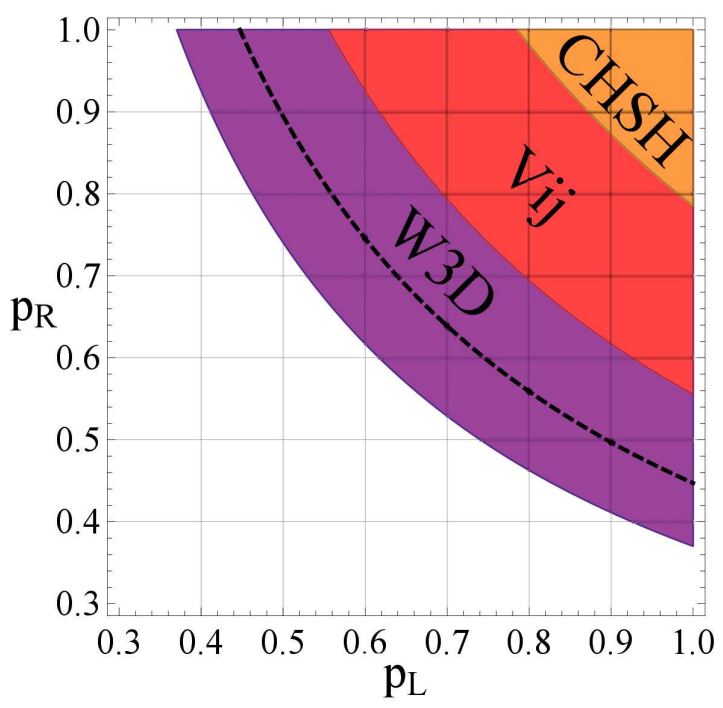

Fig. 2. Minimal required spin polarizations $p_{A}$ and $p_{B}$ to detect entangled state with the EWs and the CHSH inequality. Value of visibility parameter is fixed $\lambda=$ 0.9. Dashed line denotes required efficiency for SIMCAP protocol..

We also discuss requirements for the spin polarization of two detectors in case of some basic quantum cryptography algorithm. An example of quantum cryptography protocols that requires specific efficiency of detector to preform it are the SIngle-copy Measurement and ClAsical Processing (SIMCAP) protocols [6]. Minimal efficiency required to preform this protocol is given by:

$$
p_{A} p_{B}>\frac{1}{\sqrt[2]{5}} \approx 0.45
$$

it is clear that bound is lower than for CHSH inequalities, but greater than EWs in 3-D and 2-D.

\section{Summary}

We have discussed general properties and applications of nonideal ferromagnetic detectors in measurement of the entanglement witness operator, and compared it with the CHSH inequalities approach. Detectors described by POVM operators were introduced in order to consider non-ideal spin measurements. It can be found that EWs requires lower spin polarizations of detector to detect entanglement, and obtained state can be considered entangled and useful in performing some quantum algorithms. However, there are cryptographic algorithms which require higher efficiencies of detector in order to preform it (eg. SIMCAP protocols). Quantum description of nonideal measurements was discussed and applied to introduce a noise into the characteristics of EWs. Asymmetric spin polarizations of detectors was introduced into the system. We have concluded that in case of non-ideal detectors, only one of the detectors have to be highly spin polarized, shown in Fig. 2.

\section{Acknowledgments}

This work has been supported by the EUFP7 project SE2ND [271554] and Polish Grant for Science for the years 2010-2015.

\section{References}

[1] A. Aspect, P. Grangier, P. Gérard, R. Gérard, Phys. Rev. Lett. 47, 460 (1981).

[2] M. Horodecki, P. Horodecki, R. Horodecki, Phys. Lett. A 223, 1 (1996).

[3] M. Horodecki, P. Horodecki, R. Horodecki, Phys. Lett. A 200, 1 (1995).

[4] J. Eldridge, M.G. Pala, M. Governale, J. König, Phys. Rev. B. 82, 184507 (2010).

[5] W. Kłobus, A. Grudka, A. Baumgartner, D. Tomaszewski, C. Schönenberger, J. Martinek, Phys. Rev. B. 89, 125404 (2014).

[6] A. Acin, J. Bae, E. Bagan, M. Baig, Ll. Masanes, R. Munoz-Tapia Phys. Rev. A 73, 012327 (2006). 